\title{
RANGES OF LYAPUNOV TRANSFORMATIONS FOR OPERATOR ALGEBRAS
}

\author{
by J. KYLE
}

(Received 1 December, 1976)

1. Introduction. In this paper we shall extend results obtained in [5] to the $\mathbf{W}^{*}$-algebra setting.

Let $\mathscr{A}$ be a $C^{*}$-algebra and let $\mathscr{A}^{+}$denote the set of positive elements in $\mathscr{A}$. Given a fixed element $A$ in $\mathscr{A}$, the Lyapunov transformation $L_{A}$ corresponding to $A$ is the mapping of $\mathscr{A}$ into itself which sends $X$ to $A X+X A^{*}$. We are interested in characterizing those $B$ in $\mathscr{A}$ for which $L_{B}\left(\mathscr{A}^{+}\right)=L_{A}\left(\mathscr{A}^{+}\right)$.

Loewy in [6] and [7] examined the case when $\mathscr{A}$ is the algebra of all $n \times n$ complex matrices, and in [5] the case when $\mathscr{A}=L(H)$, for any Hilbert space $H$, was treated. As in [5], [6] and [7] we shall concentrate on non-singular Lyapunov transformations, and throughout this paper $L_{A}$ and $L_{B}$ will always be assumed to be invertible. Proof of the following may be found in [4].

Proposition 1.1. Let $A$ belong to the $W^{*}$-algebra $\mathscr{A}$. Then $L_{A}$ has a bounded inverse if and only if the spectrum of $A$ does not intersect the imaginary axis.

Let $\mathscr{A}=L(H)$ for some Hilbert space $H$. Then the main result of [5] is that the following are equivalent:

(i) $L_{A}\left(\mathscr{A}^{+}\right)=L_{B}\left(\mathscr{A}^{+}\right)$,

(ii) $B=\left(a_{1}+i a_{2} A\right)\left(a_{3} A+i a_{4}\right)^{-1}$, where $a_{i}$ are real scalars with $a_{1} a_{3}+a_{2} a_{4}=1$.

In this paper we will show that a similar result holds when $\mathscr{A}$ is any $\mathrm{W}^{*}$-algebra, and the scalars $a_{i}$ are replaced by appropriate central elements of $\mathscr{A}$. Before examining the general $\mathrm{W}^{*}$-algebra, we will show that exactly the same equivalence holds for irreducible $C^{*}$-algebras.

2. The irreducible $C^{*}$-case. Let $\mathscr{A}$ be any $\mathrm{C}^{*}$-algebra, and let $U$ denote the universal representation of $\mathscr{A}$, with $H$ denoting the Hilbert space on which $U$ acts. It is known that the second dual $U(\mathscr{A})^{* *}$ of $U(\mathscr{A})$ is a $\mathrm{W}^{*}$-algebra, and as such is isomorphic to the weak closure $\overline{U(\mathscr{A})}$ of $U(\mathscr{A})$ in $L(H)$. A proof of this may be found in [8]. However, for our purposes it is sufficient to notice that the map $\Phi$ which implements this isomorphism is obtained as follows.

For $F$ in $U(\mathscr{A})^{* *}$ and $f$ in $U(\mathscr{A})^{*}$ we know that $f=\omega_{x, y}$ for some $x, y$ in $H$. $\left(\omega_{x, y}(X)=\langle X x, y\rangle\right.$.) Hence the map $(x, y) \rightarrow F(f)=F\left(\omega_{x, y}\right)$ defines a sesquilinear form on $H$, and so by the Riesz representation of such forms, there is a unique bounded linear operator $T_{F}$ such that $F\left(\omega_{x, y}\right)=\left\langle T_{F} x, y\right\rangle$. That $T_{F}$ lies in $\overline{U(\mathscr{A})}$ is established via the double commutant theorem.

It follows easily from this that the positive cone in $U(\mathscr{A})^{* *}$ is precisely the second 
dual cone of $U(\mathscr{A})^{+}$, and that $\Phi(\hat{U(X))}=U(X)$ for all $X$ in $\mathscr{A}$. (Here denotes the canonical map into the second dual.)

Furthermore, routine calculations with the Arens' products show that

$$
\left(U \circ L_{A} \circ U^{-1}\right)^{* *}=L_{U(A)}
$$

and so

$$
\Phi\left(U \circ L_{A} \circ U^{-1}\right)^{* *} \Phi^{-1}=\bar{L}_{U(\mathrm{~A})} .
$$

(The bar indicates the natural extension of $L_{U(A)}$ to $\overline{U(\mathscr{A})}$.) Having established this notation, the next lemma follows easily.

Lemma 2.1. Suppose $A$ and $B$ belong to the $C^{*}$-algebra $A$ and that $U$ is the universal representation of $\mathscr{A}$. Then if $L_{A}\left(\mathscr{A}^{+}\right)=L_{B}\left(\mathscr{A}^{+}\right)$we also have

$$
\bar{L}_{U(A)}\left(\overline{U(\mathscr{A})}^{+}\right)=\bar{L}_{U(B)}\left(\overline{U(\mathscr{A})}^{+}\right) .
$$

Proof. Clearly by the preceding remarks

$$
\bar{L}_{U(\bar{A})}\left(\overline{U(\mathscr{A})^{+}}\right)=\bar{L}_{U(B)}\left(\overline{U(\mathscr{A})}^{+}\right)
$$

if and only if

$$
L_{U^{\prime}(A)}\left(U(\mathscr{A})^{* *+}\right)=L_{U,(B)}\left(U(\mathscr{A})^{* *+}\right) .
$$

Now suppose that $F \in U(\mathscr{A})^{* *+}, G \in U(\mathscr{A})^{* *}$ and $L_{U^{(A)}}(G)=L_{U(B)}(F)$. Then $L_{U(A)}^{* *}(G)=$ $L_{U(B)}^{* *}(F)$ and so $G\left(L_{U(A)}^{*}(f)\right)=F\left(L_{U(B)}^{*}(f)\right)$, for any $f$ in $U(\mathscr{A})^{*}$. Thus if $\omega_{x}\left(=\omega_{x, x}\right)$ is any positive functional in $U(\mathscr{A})^{*}$,

$$
G\left(\omega_{x}\right)=F\left(L_{U(B)}^{*} L_{U(A)}^{*-1}\left(\omega_{x}\right)\right) .
$$

Finally since $L_{A}\left(\mathscr{A}^{+}\right)=L_{B}\left(\mathscr{A}^{+}\right)$, we see that $L_{U(B)} L_{U(A)}^{-1}$ maps $U(\mathscr{A})^{+}$onto $U(\mathscr{A})^{+}$, and so $L_{U(B)}^{*} L_{U(A)}^{*-1}\left(\omega_{x}\right)$ is also a positive functional. Thus $F\left(L_{U(B)}^{*} L_{U(A)}^{*-1}\left(\omega_{x}\right)\right) \geqslant 0$ and so $G$ lies in $U(\mathscr{A})^{* *+}$, as required.

Proposition 2.2 Let $\mathscr{A}$ be a $C^{*}$-algebra, and let $\pi$ be any ${ }^{*}$-representation of $\mathscr{A}$. Suppose that $L_{A}\left(\mathscr{A}^{+}\right)=L_{B}\left(\mathscr{A}^{+}\right)$. Then

$$
L_{\pi(\mathrm{A})}\left(\overline{\pi(\mathscr{A})}^{+}\right)=L_{\pi(B)}\left(\overline{\pi(\mathscr{A})}^{+}\right) .
$$

Proof. If $U$ is the universal representation of $\mathscr{A}$, we can apply Lemma 2.1 to conclude that $\bar{L}_{U(A)}\left(\overline{U(\mathscr{A})^{+}}\right)=\bar{L}_{U(B)}\left(\overline{U(\mathscr{A})^{+}}\right)$. Furthermore, given any ${ }^{*}$-representation $\pi$, we can find a W*-isomorphism $\alpha$ of $\overline{\pi(A)}$ onto $\overline{U(A)} Q$, where $Q$ is some projection in the centre of $\overline{U(\mathscr{A})}$, such that $\alpha(\pi(X))=U(X) Q$ for all $X$ in $\mathscr{A}$. Now if $L_{U(A) Q}$ denotes the "cut-down" map of $\bar{L}_{U(A)}$ to the algebra $\overline{U(\mathscr{A})} Q$ (i.e. the map which sends $X Q$ to $\left(U(A) X+X U(A)^{*}\right) Q$, for all $X$ in $\overline{U(\mathscr{A}))}$, we see that $L_{U(A) Q}\left(\overline{U(\mathscr{A})}^{+} Q\right)=$ $\left.L_{U(B) Q}(\overline{U(A)})^{+} Q\right)$. Finally, since $L_{\pi(A)}=\alpha^{-1} L_{U(A) Q} \alpha$ and $L_{\pi(B)}=\alpha^{-1} L_{U(B) Q^{\alpha}}$, we con-

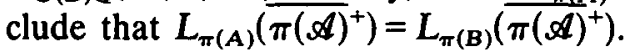


Corollary 2.3. Let $\mathscr{A}$ be a unital $C^{*}$-algebra which has a faithful irreducible *-representation. Then the following are equivalent:

(i) $L_{A}\left(\mathscr{A}^{+}\right)=L_{B}\left(\mathscr{A}^{+}\right)$,

(ii) $B=\left(a_{1}+i a_{2} A\right)\left(a_{3} A+i a_{4}\right)^{-1}$ for some real scalars $a_{i}$ with $a_{1} a_{3}+a_{2} a_{4}=1$.

Proof. Let $\pi$ be any faithful irreducible ${ }^{*}$-representation of $\mathscr{A}$ and let $H_{\pi}$ denote the Hilbert space on which $\pi$ acts. Then if $L_{A}\left(\mathscr{A}^{+}\right)=L_{B}\left(\mathscr{A}^{+}\right)$, it follows from Proposition 2.2 that $L_{\pi(A)}\left(\overline{\left.\pi(\mathscr{A})^{+}\right)}=L_{\pi(B)}\left(\overline{\pi(\mathscr{A})}^{+}\right)\right.$i.e. $L_{\pi(A)}\left(L\left(H_{\pi}\right)^{+}\right)=L_{\pi(B)}\left(L\left(H_{\pi}\right)^{+}\right)$(since $\pi$ is irreducible). Thus, by Theorem 3.2 of [5], we can find real scalars $a_{i}$ such that $a_{1} a_{3}+a_{2} a_{4}=1$ and

$$
\pi(B)=\left(a_{1}+i a_{2} \pi(A)\right)\left(a_{3} \pi(A)+i a_{4}\right)^{-1} .
$$

Now (ii) follows since $\pi$ is faithful.

Conversely, if $B$ satisfies (ii), then by the same theorem quoted above

$$
L_{\pi(A)}\left(L\left(H_{\pi}\right)^{+}\right)=L_{\pi(B)}\left(L\left(H_{\pi}\right)^{+}\right)
$$

and, since $L_{\pi(A)}^{-1} L_{\pi(B)}$ maps $\pi(\mathscr{A})$ onto itself, (i) follows, again since $\pi$ is faithful.

3. The $\mathbf{W}^{*}$-case. In this section $\mathscr{A}$ will denote a $\mathrm{W}^{*}$-algebra with centre $Z$. $\Omega$ will denote the maximal ideal space of $Z$. For any $\omega$ in $\Omega$ let $J(\omega)$ denote the smallest norm-closed two sided ideal in $\mathscr{A}$ which contains $\omega$. $\mathscr{A}(\omega)$ will denote the quotient $C^{*}$-algebra $\mathscr{A} / J(\omega)$, and $A(\omega)$ will denote the image of $A$ in $\mathscr{A}(\omega)$.

It has been shown in [2] that

$$
\|A\|=\sup \{\|A(\omega)\|: \omega \in \Omega\}
$$

and that the mapping $\omega \rightarrow\|A(\omega)\|$ is continuous. Also

$$
\operatorname{Sp}(A)=\bigcup\{\operatorname{Sp}(A(\omega)): \omega \in \Omega\} .
$$

$(\mathrm{Sp}(\cdot)$ denotes the spectrum. A proof may be found in [4].)

Before proving the main result, we need a bound for the scalars.

LEMMA 3.1. Let $A, B$ in $\mathscr{A}$ implement the Lyapunov transformations $L_{A}$ and $L_{B}$. Suppose that for each $\omega$ in $\Omega$ there are real scalars $a_{i}(\omega)$ such that $a_{1}(\omega) a_{3}(\omega)+a_{2}(\omega) a_{4}(\omega)=1$ and

$$
B(\omega)=\left[a_{1}(\omega)+i a_{2}(\omega) A(\omega)\right]\left[a_{3}(\omega) A(\omega)+i a_{4}(\omega)\right]^{-1} .
$$

Then the $a_{i}(\omega)$ are uniformly bounded by some number $K$, which depends only on $A$ and $B$.

Proof. First notice that since $A(\omega)$ and $B(\omega)$ commute, we can find a maximal abelian subalgebra $C(\omega)$ of $\mathscr{A}(\omega)$ in which both lie. Also $\operatorname{Sp}(A(\omega))$ and $\operatorname{Sp}(B(\omega))$ remain unaltered by passing to $C(\omega)$.

For any multiplicative linear functional $\phi$ on $C(\omega)$, let $a=\phi(A(\omega))$ and $b=\phi(B(\omega))$. Write $a=x_{1}+i x_{2}$ and $b=y_{1}+i y_{2}\left(x_{i}, y_{i} \in \mathbb{R}\right)$. Clearly

$$
a_{1}(\omega)+i a_{2}(\omega) a=b\left(a_{3}(\omega) a+i a_{4}(\omega)\right)
$$


and so

$$
\left[a_{1}(\omega)+i a_{2}(\omega) a\right]\left[a_{3}(\omega) \bar{a}-i a_{4}(\omega)\right]=b\left|a_{3}(\omega) a+i a_{4}(\omega)\right|^{2},
$$

from which we see that

$$
a_{1}(\omega) a_{3}(\omega) x_{1}+a_{2}(\omega) a_{4}(\omega) x_{1}=y_{1}\left|a_{3}(\omega) a+i a_{4}(\omega)\right|^{2},
$$

which reduces to

$$
x_{1}=y_{1}\left|a_{3}(\omega) a+i a_{4}(\omega)\right|^{2} .
$$

From here it is a routine matter to show that

$$
\begin{gathered}
\left|a_{3}(\omega)\right| \leqslant\left(\left|x_{1}\right| \cdot\left|y_{1}\right|\right)^{-1 / 2} \\
\left|a_{4}(\omega)\right| \leqslant\left(\left|x_{1}\right| \cdot\left|y_{1}\right|^{-1}\right)^{1 / 2}+\left(\left|x_{1}\right| \cdot\left|y_{1}\right|\right)^{-1 / 2}\left|x_{2}\right| .
\end{gathered}
$$

Using (3) and (4) we obtain

$$
\begin{gathered}
\left|a_{1}(\omega)+i a_{2}(\omega) a\right| \leqslant|b|\left(\left|x_{1}\right| \cdot\left|y_{1}\right|^{-1}\right)^{1 / 2} \\
\left|a_{2}(\omega)\right| \leqslant|b|\left(\left|x_{1}\right| \cdot\left|y_{1}\right|\right)^{-1 / 2} \\
\left|a_{1}(\omega)\right| \leqslant|b|\left\{\left(\left|x_{1}\right| \cdot\left|y_{1}\right|^{-1}\right)^{1 / 2}+\left(\left|x_{1}\right| \cdot\left|y_{1}\right|\right)^{-1 / 2}\left|x_{2}\right|\right\} .
\end{gathered}
$$

Now in the formulae (5)-(8), where $\left|x_{i}\right|$ appears without inversion, we may replace it with the spectral radius of $A(\omega)$ and so by $\|A(\omega)\|$, without disturbing the inequalities. Finally (1) shows that we may also substitute $\|A\|$. Similarly $\left|y_{1}\right|$ (but not, of course, $\left|y_{1}\right|^{-1}$ ) may be replaced by $\|B\|$, and in (7) and (8), $|b|$ may be replaced by $\|B\|$.

It remains to find upper bounds for $\left|x_{1}\right|^{-1}$ and $\left|y_{1}\right|^{-1}$. Since these represent the real parts of points in the spectra of $A(\omega)$ and $B(\omega)$, Proposition 1.1 together with (2) shows that $\left|x_{1}\right|$ and $\left|y_{1}\right|$ are bounded below by some positive number $\delta$ (which depends only on $A$ and $B$ ). Thus we may substitute $\delta^{-1}$ for $\left|x_{1}\right|^{-1}$ and $\left|y_{1}\right|^{-1}$ in (5) ... (8), and maintain the inequalities. In this way we can find a uniform bound for the scalars $a_{i}(\omega)$.

We are now in a position to prove our main result.

Theorem 3.2. Let $A$ and $B$ belong to the $W^{*}$-algebra $A$. Then the following are equivalent:

(i) $L_{A}\left(\mathscr{A}^{+}\right)=L_{B}\left(\mathscr{A}^{+}\right)$,

(ii) $B=\left(Z_{1}+i A Z_{2}\right)\left(A Z_{3}+i Z_{4}\right)^{-1}$,

where $Z_{i}$ are self-adjoint elements of the centre of $\mathscr{A}$ satisfying $Z_{1} Z_{3}+Z_{2} Z_{4}=I$.

Proof. (i) $\Rightarrow$ (ii). Clearly $L_{A}$ and $L_{B}$ map each ideal $J(\omega)$ into itself and so induce the Lyapunov transformations $L_{A(\omega)}$ and $L_{B(\omega)}$ on each $\mathscr{A}(\omega)$. Thus we see that if (i) holds, then $L_{A(\omega)}\left(\mathscr{A}(\omega)^{+}\right)=L_{B(\omega)}\left(\mathscr{A}(\omega)^{+}\right)$for each $\omega$ in $\Omega$. Also it follows from Proposition 1.1 and (2) that these induced Lyapunov transformations are non-singular.

Now in [3] Halpern has shown that each $\mathscr{A}(\omega)$ is a primitive $C^{*}$-algebra. Thus each $\mathscr{A}(\omega)$ has a faithful irreducible ${ }^{*}$-representation. Corollary 2.3 then shows that we can find real scalars $a_{i}(\omega)$ with $a_{1}(\omega) a_{3}(\omega)+a_{2}(\omega) a_{4}(\omega)=1$ such that

$$
B(\omega)=\left[a_{1}(\omega)+i a_{2}(\omega) A(\omega)\right]\left[a_{3}(\omega) A(\omega)+i a_{4}(\omega)\right]^{-1} .
$$


Lemma 3.1 shows that we can also find a constant $K$, independent of $\omega$, such that $\left|a_{i}(\omega)\right| \leqslant K$ for $i=1, \ldots, 4$, and $\omega$ in $\Omega$.

We now consider the set

$\Sigma=\left\{(\omega, a, b, c, d): B(\omega)=[a+i b A(\omega)][c A(\omega)+i d]^{-1} ; a c+b d=1\right.$ and $a, b, c, d$ bounded by $K\}$.

Then $\Sigma$ is a non-empty subset of $\Omega \times[-K, K]^{4}$ whose projection onto the first coordinate is $\Omega$.

Also $\Sigma$ is closed. For suppose $\left(\omega_{i}, a_{i}, b_{i}, c_{i}, d_{i}\right)$ is a net in $\Sigma$ which converges to $(\omega, a, b, c, d)$ in $\Omega \times[-K, K]$. Then

$$
\begin{aligned}
& \left\|B(\omega)-[a+i b A(\omega)][c A(\omega)+i d]^{-1}\right\| \\
& \quad=\left\|\left[B-(a+i b A)(c A+i d)^{-1}\right](\omega)\right\| \\
& \quad=\lim _{i}\left\|\left[B-(a+i b A)(c A+i d)^{-1}\right]\left(\omega_{i}\right)\right\| \\
& \quad=\lim _{i}\left\|B\left(\omega_{i}\right)-\left(a_{i}+i b_{i} A\left(\omega_{i}\right)\right)\left(c_{i} A\left(\omega_{i}\right)+i d_{i}\right)^{-1}\right\|=0 ;
\end{aligned}
$$

i.e. $(\omega, a, b, c, d)$ lies in $\Sigma$. Thus $\Sigma$ is a non-empty compact Hausdorff space.

Let $p_{i}$ denote the projection onto the $i$ th coordinate. Then $p_{i}$ is a continuous map of $\Sigma$ onto $\Omega$ and, since $\Omega$ is extremally disconnected, we may appeal to [1] to find a continuous selection for $p_{1}$. That is, we can find a continuous function $g$ mapping $\Omega$ into $\Sigma$ such that $p_{1} \circ g(\omega)=\omega$ for all $\omega$ in $\Omega$. (In [1] Gleason shows that in the category of all compact Hausdorff spaces and all continuous maps, the projective objects are precisely the extremally disconnected spaces.)

Thus each $Z_{i-1}=p_{i} \circ g(i=2, \ldots, 5)$ defines a continuous bounded real-valued function on $\Omega$, and so defines a self-adjoint element of $Z$. Clearly, it follows from our choice of $\Sigma$ that $B(\omega)=\left[Z_{1}+i A Z_{2}\right]\left[A Z_{3}+i Z_{4}\right]^{-1}(\omega)$ and $\left(Z_{1} Z_{3}+Z_{2} Z_{4}\right)(\omega)=1$, for all $\omega$ in $\Omega$. Thus $B=\left(Z_{1}+i A Z_{2}\right)\left(A Z_{3}+i Z_{4}\right)^{-1}$ with $Z_{1} Z_{3}+Z_{2} Z_{4}=I$ as required.

(ii) $\Rightarrow$ (i). Suppose $A$ and $B$ are related as in (ii). Then $A(\omega)$ and $B(\omega)$ are related as in (ii) of Corollary 2.3, and so $L_{A(\omega)}\left(\mathscr{A}(\omega)^{+}=L_{B(\omega)}\left(\mathscr{A}(\omega)^{+}\right)\right.$for all $\omega$ in $\Omega$. Thus if $H$ is in $\mathscr{A}^{+}$and $K=L_{A}^{-1} L_{B}(H)$ we have $K(\omega)=L_{A(\omega)}^{-1} L_{B(\omega)}(H(\omega)) \geqslant 0$ for all $\omega$ in $\Omega$; i.e. $K \geqslant 0$. Similarly $L_{B}^{-1} L_{A}\left(\mathscr{A}^{+}\right) \subseteq \mathscr{A}^{+}$and (i) follows.

Since a $C^{*}$-algebra may have no centre at all, there can be no direct generalization of Theorem 3.2 in that direction. Nonetheless we can prove the following result.

Corollary 3.3. Let $A$ and $B$ belong to the $C^{*}$-algebra $\mathscr{A}$, and suppose that $L_{A}\left(\mathscr{A}^{+}\right)=$ $L_{B}\left(\mathscr{A}^{+}\right)$. Then if $\pi$ is any ${ }^{*}$-representation of $\mathscr{A}$ we can find self-adjoint elements $Z_{i}$ in the centre of $\overline{\pi(A)}$ such that

$$
\pi(B)=\left(Z_{1}+i \pi(A) Z_{2}\right)\left(\pi(A) Z_{3}+i Z_{4}\right)^{-1} .
$$

Proof. This follows easily from Proposition 2.2 and Theorem 3.2. 


\section{REFERENCES}

1. A. M. Gleason, Projective topological spaces, Illinois J. Math. 2 (1958) 482-489.

2. J. G. Glimm, A Stone-Weierstrass theorem for $C^{*}$-algebras, Ann. of Math. 72 (1960) 216-244.

3. H. Halpern, Irreducible module homomorphisms of a von Neumann algebra into its centre, Trans. Amer. Math. Soc. 140 (1969) 195-221.

4. J. Kyle, Norms, spectra and numerical ranges of derivations, Ph.D thesis, University of Newcastle upon Tyne (1974). $99-101$.

6. R. Loewy, On ranges of Lyapunov transformations IV, Glasgow Math. J., 17 (1976) $112-118$.

7. R. Loewy, On ranges of Lyapunov transformations III, SIAM J. Appl. Math., 30 (1976) 687-702.

8. Z. Takeda, Conjugate spaces of operator algebras, Proc. Japan. Acad. 28 (1954) 90-95.

Department of Mathematics,

UNIVERSITY OF TECHNOLOGY,

LOUGHBOROUGH.

Present AdDRess

Department of Pure Mathematics,

UNIVERSITY OF BIRMINGHAM,

P.O. Box 363,

BIRMINGHAM B15 277. 